\title{
A pathological study of the lungs and heart in fatal and non-fatal chronic airways obstruction
}

\author{
K. W. M. S CO T T \\ Academic Division of Pathology, University of Sheffield
}

\begin{abstract}
Scott, K. W. M. (1976). Thorax, 31, 70-79. A pathological study of the lungs and heart in fatal and non-fatal chronic airways obstruction. The lungs and hearts from 50 patients were examined using morphometric techniques to determine the size of the right ventricle, the amount, type, and distribution of emphysema, the size of the bronchial mucous glands, and the proportion of the lung occupied by small airways of less than $2 \mathrm{~mm}$ diameter. The patients were divided into three groups according to the clinical history: 18 died as a result of chronic airways obstruction, 17 had symptoms of chronic chest disease but died from some unrelated cause, and 15 had no symptoms related to the respiratory system. The total amount of emphysema was found to be greater in the fatal than the symptomatic group who in turn had more emphysema than the asymptomatic group. A positive correlation was found between the amount of emphysema and the right ventricular weight. The amount of panlobular emphysema in the lung was found to be greater in the fatal group than in the others but this did not apply to the amount of centrilobular emphysema. The amount of panlobular, but not centrilobular, emphysema showed a positive correlation with right ventricular weight. As the total amount of emphysema increased it was found that there was an increase in each of three zones in the lung-apical, middle, and lower. There was no relationship between the bronchial mucous gland size and either the clinical state of the patients with symptoms or the right ventricular weight. The proportion of lung occupied by the lumen of small airways was significantly reduced in the fatal group as compared to the other two groups and also showed a negative (inverse) correlation with right ventricular weight.

The total amount of emphysema, the amount of panlobular emphysema, and reduction in small airways lumen in the lung are the three factors in chronic airways obstruction which are quantitatively related to death in chronic airways obstruction and to right ventricular weight.
\end{abstract}

Several studies have been carried out on the relationship between death in heart failure, following chronic airways obstruction, and the amount and type of emphysema in the lung at necropsy (Sweet et al., 1961; Burrows et al., 1966). There have also been studies of the relationship between right ventricular weight and the amount and type of emphysema (Cromie, 1961; Hicken, Heath, and Brewer, 1966; James, 1966; Hasleton, 1973). Bignon et al. (1969) and Bignon, AndreBougaran, and Brouet (1970) examined the relationship between other elements of chronic airways obstruction, such as bronchial mucous gland size in large airways and the number and size of small airways, and right ventricular weight.
The aim of the present study was to determine whether there were morphological differences in chronic airways obstruction between patients dying as a result of the disease and patients whoN have chronic chest disease yet die from some ${ }_{\sigma}^{\omega}$ unrelated cause, not having developed any complications. It was decided to examine, morphometrically, the three main components of thes chronic airways obstruction complex-the bronchial mucous gland size as evidence of large air- 0 ways disease; the proportion of small airways of less than $2 \mathrm{~mm}$ diameter lumen in the lung as an index of small airways disease; the amount, type, and distribution of emphysema in the lung, reflecting damage to the alveolar sacs. As well as 
relating these parameters to the clinical state of the patient they were also compared to the weight of the right ventricle.

\section{MATERIAL AND METHODS}

The investigation was carried out on hearts and left lungs, obtained at necropsy, from 50 patients. Clinical details of the patients were obtained from the case records and this permitted the division of the 50 cases into three separate groups. The first group consisted of 15 cases where there was no history of cough, sputum production or breathlessness, and death was not due to respiratory disease. The second group consisted of 18 patients who died as a result of chronic airways obstruction. The majority had heart failure, for which no cause other than lung disease could be found and many of them had a terminal acute bronchitis. The clinical findings in this group of patients will be considered in a future study. The third group consisted of 17 patients who had a history of cough and sputum production consistent with a clinical diagnosis of chronic bronchitis. They had never complained of breathlessness, and all the patients in this group died from conditions other than chronic airways obstruction.

The hearts were fixed, following examination of the coronary arteries, in $10 \%$ formol saline for 72-96 hours and were then dissected according to the method of Fulton, Hutchinson, and Jones (1952). The right ventricle and left ventricle plus the septum were weighed separately, and cases which showed evidence of left ventricular hypertrophy (left ventricle plus septum weighed more than $225 \mathrm{~g}$ ) were excluded. Also excluded were cases of systemic hypertension, gross coronary artery disease, and myocardial fibrosis.

The lungs were distended with $10 \%$ formol saline introduced by a catheter into the left main bronchus, at a pressure of $30 \mathrm{~cm}$ of water, using a modification of the apparatus designed by Heard (1969). Following fixation in this state, samples of the bronchial tree were taken from three sites: (1) the left main bronchus just proximal to its bifurcation, (2) the bronchus to the basal segments (the bronchus immediately below the origin of the bronchus to the apical segment of the lower lobe), and (3) the bronchus to the inferior segment of the lingula $5 \mathrm{~mm}$ beyond its origin (Restrepo and Heard, 1963). Transverse blocks were embedded in paraffin, and $5 \mu$ sections were stained by haematoxylin and eosin and periodic acid schiff, following which they were projected, at a magnification of 20 diameters, on to a point-counting grid (the points were at the angles of equilateral triangles of side $0.6 \mathrm{~cm}$ ) (Dunnill, Massarella, and Anderson, 1969). The number of points falling on bronchial mucous glands was expressed as a percentage of the total number of points falling on tissue, and a mean was taken of the values for each of the three sites. The lung was then sliced in the parasagittal plane at $1 \mathrm{~cm}$ intervals and, following barium sulphate impregnation (Heard, 1958), three or four slices were examined to determine the amount and type of emphysema present. This was done using a plastic grid and a point-counting technique (Dunnill, 1962). The type of tissue under each point in the grid was noted, and a count was made of the following components in each slice-panlobular emphysema (distensive and destructive), centrilobular emphysema (distensive and destructive), and normal lung and non-parenchyma (blood vessels, bronchi, pleura, etc). The amount of each type of emphysema was expressed as a percentage of the total number of points counted, and a mean was taken of the three or four slices examined. The total amount of emphysema present was also calculated as a percentage. The lung slices were also divided into three zones of equal vertical height by placing transparent plastic strips horizontally across the slices, the total height from apex to base having previously been measured. The three zones were called zone 1 (apical), zone 2 (middle), and zone 3 (lower), and the amount of emphysema in each of these three zones was recorded as a percentage of the number of points counted in that zone. Using a stratified random sampling technique (Dunnill, 1968a), six blocks of approximately $2.5 \times 1.5 \times 0.5 \mathrm{~cm}$ were selected from the lung slices. These were embedded in paraffin, sectioned, and stained by haematoxylin and eosin. The relative proportion of small airways of diameter less than $2 \mathrm{~mm}$ was calculated using an eyepiece graticule with 25 equidistant points. Each point was scored as either small airways lumen or other lung tissue, and the proportion of small airways lumen (Q) in the lung was expressed as a percentage of the total number of points counted (Matsuba and Thurlbeck, 1971). It was assumed that shrinkage due to fixation and processing was equal in small airways and in the remainder of the lung tissue, and $Q$ was not corrected for the degree of inflation as Matsuba and Thurlbeck (1972) found this not to be of major significance.

\section{RESULTS}

Table I shows the correlation coefficients for all 50 cases, irrespective of clinical status, when each 
T A B L E I

CORRELATION COEFFICIENTS BETWEEN EACH PARAMETER MEASURED AND SIZE OF RIGHT VENTRICLE (WEIGHT AND LV/RV RATIO)

\begin{tabular}{|c|c|c|c|c|c|}
\hline \multirow[b]{2}{*}{ Parameter } & \multicolumn{2}{|c|}{ Right Ventricular Weight (g) } & \multicolumn{2}{|c|}{ Ratio of LV/RV } & \\
\hline & $\mathbf{r}$ & $\mathbf{P}$ & $\mathbf{r}$ & $\mathbf{P}$ & \\
\hline 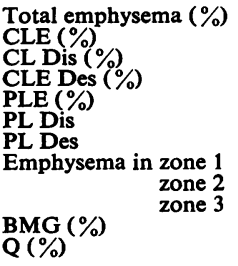 & $\begin{array}{l}+0.6325 \\
+0.0522 \\
+0.0031 \\
+0.0887 \\
+0.6448 \\
+0.6437 \\
+0.2787 \\
+0.6108 \\
+0.6574 \\
+0.6282 \\
+0.1703 \\
-0.4895\end{array}$ & $\begin{aligned} &< 0.001 \\
&> 0.10 \\
&> 0.90 \\
&> 0.50 \\
&< 0.001 \\
&<0.001 \\
& 0.05 \\
&<0.001 \\
&<0.001 \\
&<0.001 \\
&>0.10 \\
&<0.001\end{aligned}$ & $\begin{array}{l}-0.6838 \\
-0.0397 \\
-0.0333 \\
-0.2007 \\
-0.6248 \\
-0.4918 \\
-0.4389 \\
-0.6846 \\
-0.6488 \\
-0.5947 \\
-0.2172 \\
+0.4189\end{array}$ & $\begin{array}{l}<0.001 \\
>0.10 \\
>0.10 \\
>0.10 \\
<0.001 \\
<0.001 \\
<0.01 \\
<0.001 \\
<0.001 \\
<0.001 \\
>0.10 \\
<0.10\end{array}$ & $\begin{array}{l}\text { s } \\
\text { ns } \\
\text { ns } \\
\text { ns } \\
\text { s } \\
\text { s】l } \\
\text { s } \\
\text { s]l } \\
\mathbf{s} \\
\text { s } \\
\text { ns } \\
\text { s }\end{array}$ \\
\hline
\end{tabular}

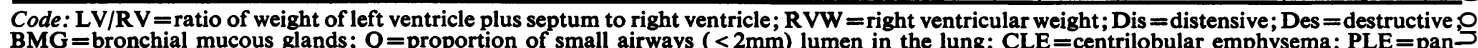
lobular emphysema; $L V=$ left ventricle plus septum; $R V=$ right ventricle; $s=$ statistically significant; ns=not statistically significant.

parameter was compared to the right ventricular weight. There was a significant positive correlation between right ventricular weight and the total amount of emphysema present, as shown in Figure 1. No significant correlation was found between the amount of centrilobular emphysema and right ventricular weight but there was a significant correlation between the amount of panlobular emphysema and right ventricular weight. This applied both to the total amount and to both types of panlobular emphysema. Also the amount of emphysema in each of the three zones increased significantly with right ventricular weight. There was no correlation between the bronchial mucous gland size and right ventricular weight but there was a significant negative correlation between the

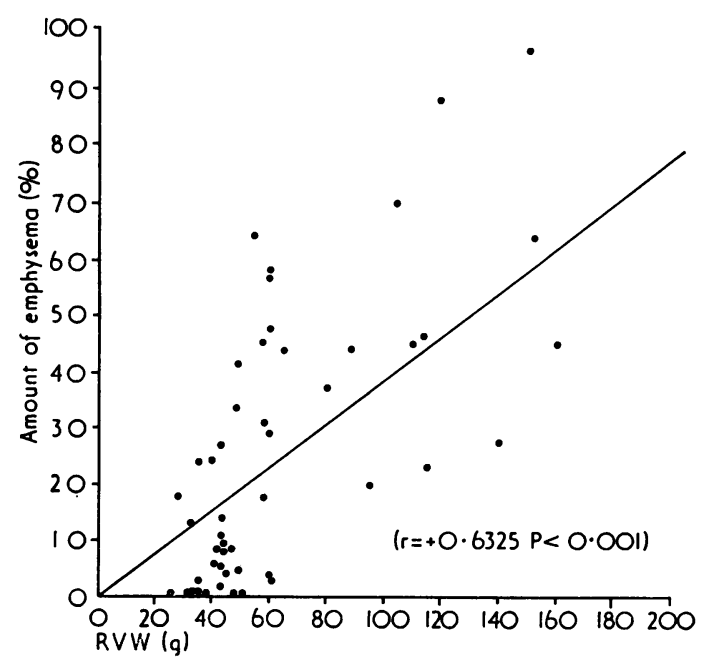

FIG. 1. Relationship between total amount of emphysema and right ventricular weight.

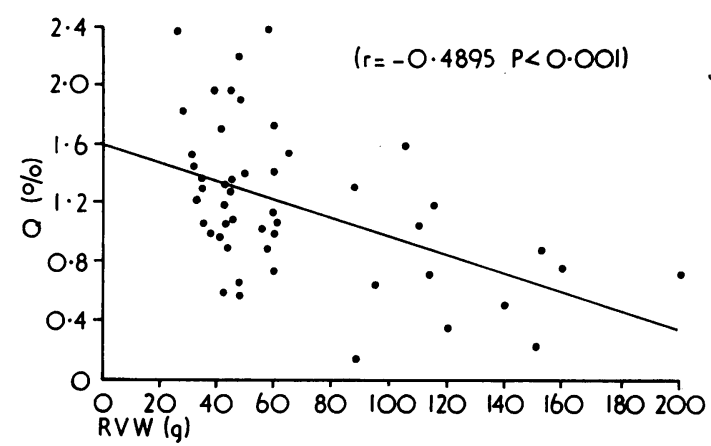

FIG. 2. Relationship between proportion of smallo airways lumen $(Q)$ in the lung and right ventricular weight.

proportion of small airways lumen and right ven-o tricular weight, as shown in Figure 2. Correlation? coefficients were also calculated for the relation- $\varrho$ ship between the same parameters and the ratio of $₹$ the weight of left ventricle plus septum to the의 right ventricle. These are also shown in Table I. $>$ The results are the same as for the weight of the right ventricle with the exception that positiven correlations become negative correlations when the ratio is considered. Correlation coefficients $\tilde{N}$ were also calculated on the 35 cases with lung disease. There was a significant positive correla-O tion between right ventricular weight and the totako amount of emphysema $(r=0.541, P<0.001)$ and also between right ventricular weight and panlo-? bular emphysema $(r=0.561, P<0.001)$. The relationship between right ventricular weight and the proportion of small airways lumen in the lung@ was also significant but inverse $(r=-0.508, \AA$ $0.01>\mathbf{P}>0.001$ ). Table II shows several other correlation coefficients which were calculated. There 
was a significant negative correlation between the proportion of small airways lumen in the lung and both the total amount of emphysema and the amount of panlobular emphysema. No relationship between the proportion of small airways lumen and either the amount of centrilobular emphysema or the bronchial mucous gland size was found. The bronchial mucous gland size had no significant relationship with either the total amount of emphysema or the amount of centrilobular emphysema.

The results obtained in the 15 patients without respiratory symptoms are shown in Table III. This includes the right ventricular weight, the ratio of left ventricle plus septum to the right ventricle, the total amount of emphysema, the amount of centrilobular emphysema, the amount of panlobular emphysema, the distribution of the emphysema, the bronchial mucous gland size, and the proportion of small airways $(<2 \mathrm{~mm}$ diameter) lumen in the lung. The results for the 18 patients dying from chronic airways obstruction are shown in Table IV, and those for the 17 patients with non-fatal respiratory disease are given in Table $\mathrm{V}$. The mean values for each parameter measured were compared using Student's $t$ test, and a com- parison of the means of the fatal and symptomfree groups is shown in Table VI. The fatal group had a significantly greater mean right ventricular weight, a significantly greater amount of emphysema, both in total and of all types, and also a greater amount of emphysema in each of the three zones measured than the symptom-free group. The fatal group also had a significantly larger mean bronchial mucous gland size and a significantly smaller proportion of small airways lumen in the lung than the symptom-free group. Table VI shows the comparison of the mean values in the fatal and non-fatal groups with respiratory disease. The right ventricular weight was significantly greater in the fatal group, as was the total amount of emphysema. However, there was no significant difference between the total amount of centrilobular emphysema and the amounts of centrilobular distensive and destructive emphysema in the two groups. There was a significantly greater amount of panlobular emphysema in the fatal group than in the non-fatal group. This applied to the total amount of emphysema and the amount of distensive and destructive emphysema. The greater amount of emphysema in the fatal group was present in all

T A B L E I I

CORRELATION COEFFICIENTS BETWEEN SEVERAL DIFFERENT PARAMETERS ON 50 CASES

\begin{tabular}{l|c|c|c|}
\hline \multicolumn{1}{c|}{ Parameters compared } & r & P \\
\hline Q and total emphysema & -0.3880 & $>0.01$ & $>0.10$ \\
$Q$ and CLE & -0.0277 & $<0.01$ & $>0.10$ \\
$Q$ and PLE & -0.3897 & $>0.10$ & s \\
$Q$ and BMG & -0.2201 & s & ns \\
BMG and total emphysema & +0.2217 & ns & ns \\
BMG and CLE & +0.2216 & \\
\hline
\end{tabular}

For abbreviations see footnote to Table I.

T A B L E I I I

DETAILS OF 15 CASES WITHOUT RESPIRATORY SYMPTOMS

\begin{tabular}{|c|c|c|c|c|c|c|c|c|c|c|c|c|c|c|c|c|}
\hline \multirow{2}{*}{$\begin{array}{l}\text { Case } \\
\text { No. }\end{array}$} & \multirow[b]{2}{*}{ Age } & \multirow[b]{2}{*}{ Sex } & \multirow{2}{*}{$\underset{\text { (g) }}{\text { RVW }}$} & \multirow{2}{*}{$\begin{array}{l}\text { LVI } \\
\text { RV }\end{array}$} & \multirow{2}{*}{$\begin{array}{c}\text { Total } \\
\text { Emphy- } \\
\text { sema } \\
\%\end{array}$} & \multicolumn{3}{|c|}{$\begin{array}{l}\text { Centrilobular } \\
\text { Emphysema \% }\end{array}$} & \multicolumn{3}{|c|}{$\begin{array}{c}\text { Panlobular } \\
\text { Emphysema \% }\end{array}$} & \multicolumn{3}{|c|}{$\begin{array}{l}\text { Distribution of } \\
\text { Emphysema \% }\end{array}$} & \multirow[t]{2}{*}{$\underset{\%}{\text { BMG }}$} & \multirow[t]{2}{*}{ Q \% } \\
\hline & & & & & & Total & Dis & Des & Total & Dis & Des & Zone 1 & Zone 2 & Zone 3 & & \\
\hline $\begin{array}{l}2 \\
10 \\
11 \\
14 \\
17 \\
28 \\
36 \\
38 \\
40 \\
42 \\
43 \\
54 \\
55 \\
56 \\
59\end{array}$ & $\begin{array}{l}66 \\
51 \\
78 \\
82 \\
70 \\
66 \\
60 \\
17 \\
66 \\
52 \\
72 \\
54 \\
89 \\
74 \\
66\end{array}$ & $\begin{array}{l}\mathbf{M} \\
\mathbf{M} \\
\mathbf{M} \\
\mathbf{F} \\
\mathbf{F} \\
\mathbf{F} \\
\mathbf{F} \\
\mathbf{M} \\
\mathbf{M} \\
\mathbf{F} \\
\mathbf{F} \\
\mathbf{M} \\
\mathbf{F} \\
\mathbf{M} \\
\mathbf{F}\end{array}$ & $\begin{array}{l}35 \\
45 \\
60 \\
43 \\
33 \\
48 \\
38 \\
50 \\
49 \\
32 \\
26 \\
61 \\
32 \\
43 \\
43\end{array}$ & $\begin{array}{l}3 \cdot 9 \\
2 \cdot 2 \\
3 \cdot 8 \\
4 \cdot 0 \\
3 \cdot 7 \\
2 \cdot 5 \\
3 \cdot 6 \\
3 \cdot 6 \\
3 \cdot 4 \\
6 \cdot 1 \\
4 \cdot 2 \\
3 \cdot 6 \\
5 \cdot 2 \\
3 \cdot 6 \\
2 \cdot 8\end{array}$ & $\begin{array}{c}2 \cdot 0 \\
3 \cdot 8 \\
3 \cdot 0 \\
1 \cdot 4 \\
13 \cdot 0 \\
0 \\
0 \\
0 \\
4 \cdot 0 \\
0 \\
0 \\
2 \cdot 4 \\
0 \\
11 \cdot 0 \\
5 \cdot 0\end{array}$ & $\begin{array}{c}2 \cdot 0 \\
3 \cdot 8 \\
1 \cdot 75 \\
1 \cdot 4 \\
13 \cdot 0 \\
0 \\
0 \\
0 \\
4 \cdot 0 \\
0 \\
0 \\
1 \cdot 4 \\
0 \\
11 \cdot 0 \\
2\end{array}$ & $\begin{array}{l}1 \cdot 0 \\
3 \cdot 8 \\
1 \cdot 5 \\
1 \cdot 4 \\
12 \cdot 0 \\
0 \\
0 \\
0 \\
4 \cdot 0 \\
0 \\
0 \\
1 \cdot 4 \\
0 \\
8 \cdot 5 \\
2\end{array}$ & $\begin{array}{l}1 \cdot 0 \\
0 \\
0 \cdot 25 \\
0 \\
1 \cdot 0 \\
0 \\
0 \\
0 \\
0 \\
0 \\
0 \\
0 \\
0 \\
2 \cdot 5 \\
0\end{array}$ & $\begin{array}{l}0 \\
0 \\
1 \cdot 25 \\
0 \\
0 \\
0 \\
0 \\
0 \\
0 \\
0 \\
0 \\
1 \\
0 \\
0 \\
3\end{array}$ & $\begin{array}{l}0 \\
0 \\
1 \cdot 0 \\
0 \\
0 \\
0 \\
0 \\
0 \\
0 \\
0 \\
0 \\
1 \\
0 \\
0 \\
3\end{array}$ & $\begin{array}{l}\mathbf{0} \\
0 \\
0 \cdot 25 \\
0 \\
0 \\
0 \\
0 \\
0 \\
0 \\
0 \\
0 \\
0 \\
0 \\
0 \\
0\end{array}$ & $\begin{array}{r}6 \\
11 \\
2 \\
4 \\
24 \\
0 \\
0 \\
0 \\
8 \\
0 \\
0 \\
7 \\
0 \\
20 \\
14\end{array}$ & $\begin{array}{l}0 \\
0 \\
1 \\
0 \\
6 \\
0 \\
0 \\
0 \\
3 \cdot 5 \\
0 \\
0 \\
1 \\
0 \\
14 \\
0\end{array}$ & $\begin{array}{l}0 \\
0 \\
0 \\
0 \\
1 \\
0 \\
0 \\
0 \\
3 \\
0 \\
0 \\
0 \\
0 \\
0 \\
0 \\
2 \\
0\end{array}$ & $\begin{array}{r}12 \cdot 2 \\
17.3 \\
10 \cdot 6 \\
12.1 \\
11 \cdot 5 \\
12.0 \\
13.0 \\
8 \cdot 0 \\
10.0 \\
15.0 \\
6.0 \\
19.0 \\
11.2 \\
6.4 \\
14.6\end{array}$ & $\begin{array}{l}1.05 \\
1.26 \\
1.73 \\
1.31 \\
1.21 \\
0.63 \\
0.96 \\
1.96 \\
1.39 \\
1.43 \\
2.37 \\
1.06 \\
1.53 \\
1.17 \\
1.05\end{array}$ \\
\hline $\begin{array}{l}\text { Mean } \\
\text { SD }\end{array}$ & $\begin{array}{l}64 \cdot 2 \\
16 \cdot 85\end{array}$ & & $\begin{array}{l}42 \cdot 5 \\
10 \cdot 1\end{array}$ & $\begin{array}{l}3 \cdot 75 \\
0 \cdot 9\end{array}$ & $\begin{array}{l}3 \cdot 1 \\
3 \cdot 9\end{array}$ & $\begin{array}{l}2 \cdot 7 \\
4 \cdot 0\end{array}$ & $\begin{array}{l}2 \cdot 4 \\
3 \cdot 5\end{array}$ & $\begin{array}{l}0 \cdot 3 \\
2 \cdot 2\end{array}$ & $\begin{array}{l}0.3 \\
0.8\end{array}$ & $\begin{array}{l}0 \cdot 4 \\
0 \cdot 8\end{array}$ & $\begin{array}{l}0.02 \\
0.06\end{array}$ & $\begin{array}{l}6 \cdot 4 \\
7 \cdot 7\end{array}$ & $\begin{array}{l}1 \cdot 7 \\
3 \cdot 8\end{array}$ & $\begin{array}{l}0.4 \\
0.9\end{array}$ & $\begin{array}{r}11 \cdot 9 \\
3 \cdot 6\end{array}$ & $\begin{array}{l}1 \cdot 34 \\
0.43\end{array}$ \\
\hline
\end{tabular}

For abbreviations see footnote to Table I. 
T A B L E I V

DETAILS OF 18 CASES OF FATAL CHRONIC AIRWAYS OBSTRUCTION

\begin{tabular}{|c|c|c|c|c|c|c|c|c|c|c|c|c|c|c|c|c|}
\hline \multirow{2}{*}{$\begin{array}{l}\text { Case } \\
\text { No. }\end{array}$} & \multirow{2}{*}{ Age } & \multirow{2}{*}{ Sex } & \multirow{2}{*}{$\begin{array}{l}\text { RVW } \\
\text { (g) }\end{array}$} & \multirow{2}{*}{$\begin{array}{l}\text { LV/ } \\
\text { RV }\end{array}$} & \multirow{2}{*}{$\begin{array}{c}\text { Total } \\
\text { Emphy- } \\
\text { sema } \\
\%\end{array}$} & \multicolumn{3}{|c|}{$\begin{array}{l}\text { Centrilobular } \\
\text { Emphysema \% }\end{array}$} & \multicolumn{3}{|c|}{$\begin{array}{c}\text { Panlobular } \\
\text { Emphysema \% }\end{array}$} & \multicolumn{3}{|c|}{$\begin{array}{l}\text { Distribution of } \\
\text { Emphysema } \%\end{array}$} & \multirow{2}{*}{$\underset{\%}{\mathrm{BMG}}$} & \multirow{2}{*}{ 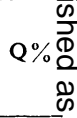 } \\
\hline & & & & & & Total & Dis & Des & Total & Dis & Des & Zone 1 & Zone 2 & Zone 3 & & \\
\hline $\begin{array}{r}7 \\
9 \\
15 \\
19 \\
20 \\
21 \\
27 \\
30 \\
34 \\
45 \\
46 \\
49 \\
50 \\
51 \\
68 \\
70 \\
72 \\
73\end{array}$ & $\begin{array}{l}60 \\
60 \\
70 \\
70 \\
60 \\
48 \\
76 \\
51 \\
60 \\
61 \\
38 \\
66 \\
64 \\
64 \\
68 \\
49 \\
68 \\
62 \\
\end{array}$ & $\begin{array}{l}\mathbf{F} \\
\mathbf{M} \\
\mathbf{F} \\
\mathbf{M} \\
\mathbf{M} \\
\mathbf{M} \\
\mathbf{M} \\
\mathbf{M} \\
\mathbf{M} \\
\mathbf{M} \\
\mathbf{F} \\
\mathbf{M} \\
\mathbf{M} \\
\mathbf{M} \\
\mathbf{M} \\
\mathbf{F} \\
\mathbf{M} \\
\mathbf{M}\end{array}$ & $\begin{array}{r}65 \\
60 \\
43 \\
105 \\
114 \\
115 \\
88 \\
152 \\
95 \\
160 \\
199 \\
140 \\
55 \\
110 \\
80 \\
120 \\
60 \\
150 \\
\end{array}$ & $\begin{array}{l}2.2 \\
2.6 \\
4.3 \\
1.6 \\
1.9 \\
1.8 \\
2.2 \\
1.4 \\
1.7 \\
1.2 \\
1.0 \\
1.5 \\
1.9 \\
1.6 \\
2.1 \\
0.9 \\
2.1 \\
1.4\end{array}$ & $\begin{array}{l}44 \cdot 0 \\
48 \cdot 0 \\
14 \cdot 0 \\
70 \cdot 0 \\
47 \cdot 0 \\
23 \cdot 0 \\
44 \cdot 0 \\
64 \cdot 0 \\
20 \cdot 6 \\
45 \cdot 0 \\
61 \cdot 0 \\
28 \cdot 0 \\
64 \cdot 0 \\
45 \cdot 0 \\
38 \cdot 0 \\
88 \cdot 0 \\
58 \cdot 0 \\
96 \cdot 0\end{array}$ & $\begin{array}{r}2 \cdot 5 \\
47 \cdot 0 \\
14 \cdot 0 \\
4 \cdot 0 \\
8 \cdot 0 \\
22 \cdot 0 \\
14 \cdot 0 \\
5 \cdot 0 \\
9 \cdot 6 \\
16 \cdot 0 \\
2 \cdot 0 \\
28 \cdot 0 \\
26 \cdot 0 \\
2 \cdot 0 \\
13 \cdot 0 \\
0 \\
0 \\
0\end{array}$ & $\begin{array}{r}0 \\
31 \cdot 0 \\
12 \cdot 0 \\
3 \cdot 0 \\
6 \cdot 0 \\
10 \cdot 0 \\
12 \cdot 0 \\
5 \cdot 0 \\
8 \cdot 0 \\
6 \cdot 0 \\
0 \cdot 5 \\
18 \cdot 0 \\
3 \cdot 0 \\
1 \cdot 0 \\
12 \cdot 0 \\
0 \\
0 \\
0 \\
\end{array}$ & $\begin{array}{r}2 \cdot 5 \\
16 \cdot 0 \\
2 \cdot 0 \\
1 \cdot 0 \\
2 \cdot 0 \\
12 \cdot 0 \\
2 \cdot 0 \\
0 \\
1 \cdot 6 \\
10 \cdot 0 \\
1 \cdot 5 \\
10 \cdot 0 \\
23 \cdot 0 \\
1 \cdot 0 \\
1 \cdot 0 \\
0 \\
0 \\
0\end{array}$ & $\begin{array}{c}41 \cdot 2 \\
1 \cdot 0 \\
0 \\
66 \cdot 0 \\
39 \cdot 0 \\
1 \cdot 0 \\
30 \cdot 0 \\
59 \cdot 0 \\
10 \cdot 7 \\
29 \cdot 0 \\
59 \cdot 0 \\
0 \\
38 \cdot 0 \\
43 \cdot 0 \\
25 \cdot 0 \\
88 \cdot 0 \\
58 \cdot 0 \\
96 \cdot 0\end{array}$ & $\begin{array}{c}1 \cdot 2 \\
0 \cdot 5 \\
0 \\
48 \cdot 0 \\
35 \cdot 0 \\
1 \cdot 0 \\
26 \cdot 0 \\
59 \cdot 0 \\
7 \cdot 5 \\
14 \cdot 5 \\
40 \cdot 0 \\
0 \\
0 \\
20 \cdot 0 \\
20 \cdot 0 \\
10 \cdot 0 \\
19 \cdot 0 \\
92 \cdot 0\end{array}$ & $\begin{array}{c}40 \cdot 0 \\
0 \cdot 5 \\
0 \\
18 \cdot 0 \\
4 \cdot 0 \\
0 \\
4 \cdot 0 \\
0 \\
3 \cdot 2 \\
14 \cdot 5 \\
19 \cdot 0 \\
0 \\
38 \cdot 0 \\
23 \cdot 0 \\
5 \cdot 0 \\
78 \cdot 0 \\
39 \cdot 0 \\
4 \cdot 0\end{array}$ & $\begin{array}{r}74 \\
57 \\
21 \\
83 \\
75 \\
31 \\
76 \\
78 \\
37 \\
72 \\
80 \\
30 \\
89 \\
84 \\
76 \\
100 \\
93 \\
97 \\
\end{array}$ & $\begin{array}{l}49 \\
52 \\
13 \\
67 \\
14 \\
21 \\
32 \\
77 \\
20 \\
56 \\
63 \\
32 \\
69 \\
54 \\
42 \\
90 \\
37 \\
90\end{array}$ & $\begin{array}{r}9 \\
36 \\
5 \\
65 \\
32 \\
16 \\
18 \\
37 \\
0 \\
18 \\
42 \\
24 \\
38 \\
11 \\
12 \\
77 \\
47 \\
90\end{array}$ & $\begin{array}{l}18 \cdot 0 \\
21 \cdot 8 \\
23 \cdot 0 \\
16 \cdot 0 \\
23 \cdot 0 \\
18 \cdot 6 \\
12 \cdot 8 \\
14 \cdot 1 \\
26 \cdot 0 \\
19 \cdot 7 \\
20 \cdot 5 \\
15 \cdot 6 \\
20 \cdot 1 \\
14 \cdot 0 \\
14 \cdot 0 \\
11 \cdot 2 \\
28 \cdot 4 \\
21 \cdot 5\end{array}$ & 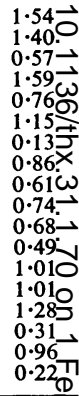 \\
\hline $\begin{array}{l}\text { Mean } \\
\text { SD }\end{array}$ & $\begin{array}{r}60 \cdot 8 \\
9 \cdot 3\end{array}$ & & $\begin{array}{r}106 \cdot 2 \\
42 \cdot 5\end{array}$ & $\begin{array}{l}1 \cdot 86 \\
0.7\end{array}$ & $\begin{array}{l}49 \cdot 3 \\
21 \cdot 2\end{array}$ & $\begin{array}{l}11 \cdot 8 \\
12 \cdot 5\end{array}$ & $\begin{array}{l}7 \cdot 1 \\
8 \cdot 0\end{array}$ & $\begin{array}{l}4 \cdot 7 \\
6 \cdot 6\end{array}$ & $\begin{array}{l}38 \cdot 0 \\
29 \cdot 3\end{array}$ & $\begin{array}{l}21 \cdot 8 \\
25 \cdot 0\end{array}$ & $\begin{array}{l}16 \cdot 1 \\
20 \cdot 9\end{array}$ & $\begin{array}{l}69 \cdot 6 \\
24 \cdot 2\end{array}$ & $\begin{array}{l}48 \cdot 7 \\
24 \cdot 3\end{array}$ & $\begin{array}{l}32 \cdot 0 \\
25 \cdot 1\end{array}$ & $\begin{array}{r}18 \cdot 8 \\
4 \cdot 7\end{array}$ & $\begin{array}{l}0.85 \\
0.43 \frac{0}{\underline{\underline{2}}}\end{array}$ \\
\hline
\end{tabular}

For abbreviations see footnote to Table I.

T A B L E V

DETAILS OF 17 CASES OF NON-FATAL RESPIRATORY DISEASE

\begin{tabular}{|c|c|c|c|c|c|c|c|c|c|c|c|c|c|c|c|c|}
\hline \multirow{2}{*}{$\begin{array}{l}\text { Case } \\
\text { No. }\end{array}$} & \multirow{2}{*}{ Age } & \multirow{2}{*}{ Sex } & \multirow{2}{*}{$\begin{array}{c}\text { RVW } \\
\text { (g) }\end{array}$} & \multirow{2}{*}{$\begin{array}{l}\text { LVI } \\
\text { RV }\end{array}$} & \multirow{2}{*}{$\begin{array}{c}\text { Total } \\
\text { Emphy- } \\
\text { sema } \\
\%\end{array}$} & \multicolumn{3}{|c|}{$\begin{array}{l}\text { Centrilobular } \\
\text { Emphysema \% }\end{array}$} & \multicolumn{3}{|c|}{$\begin{array}{c}\text { Panlobular } \\
\text { Emphysema \% }\end{array}$} & \multicolumn{3}{|c|}{$\begin{array}{l}\text { Distribution of } \\
\text { Emphysema \% }\end{array}$} & \multirow{2}{*}{$\underset{\%}{\text { BMG }}$} & \multirow{2}{*}{ Q \% } \\
\hline & & & & & & Total & Dis & Des & Total & Dis & Des & $\overline{\text { Zone } 1}$ & Zone 2 & Zone 3 & & \\
\hline $\begin{array}{r}6 \\
13 \\
18 \\
22 \\
23 \\
24 \\
31 \\
32 \\
39 \\
41 \\
47 \\
52 \\
58 \\
60 \\
63 \\
65 \\
69\end{array}$ & $\begin{array}{l}61 \\
46 \\
72 \\
69 \\
66 \\
72 \\
56 \\
69 \\
56 \\
68 \\
68 \\
60 \\
78 \\
53 \\
74 \\
70 \\
73\end{array}$ & $\begin{array}{l}\mathbf{F} \\
\mathbf{F} \\
\mathbf{M} \\
\mathbf{M} \\
\mathbf{M} \\
\mathbf{M} \\
\mathbf{M} \\
\mathbf{F} \\
\mathbf{M} \\
\mathbf{F} \\
\mathbf{M} \\
\mathbf{F} \\
\mathbf{M} \\
\mathbf{M} \\
\mathbf{M} \\
\mathbf{M} \\
\mathbf{M}\end{array}$ & $\begin{array}{l}28 \\
35 \\
48 \\
41 \\
44 \\
40 \\
58 \\
35 \\
42 \\
48 \\
45 \\
58 \\
48 \\
57 \\
45 \\
60 \\
60\end{array}$ & $\begin{array}{l}4 \cdot 2 \\
3 \cdot 3 \\
3 \cdot 1 \\
3 \cdot 5 \\
3 \cdot 5 \\
3 \cdot 5 \\
2 \cdot 4 \\
3 \cdot 6 \\
4 \cdot 3 \\
4 \cdot 6 \\
3 \cdot 4 \\
2 \cdot 7 \\
2 \cdot 7 \\
3 \cdot 5 \\
2.6 \\
3 \cdot 0 \\
3 \cdot 0\end{array}$ & $\begin{array}{r}17 \cdot 6 \\
0 \\
8 \cdot 5 \\
5 \cdot 0 \\
27 \cdot 0 \\
24 \cdot 0 \\
31 \cdot 0 \\
24 \cdot 0 \\
8 \cdot 0 \\
33 \cdot 0 \\
9 \cdot 0 \\
17 \cdot 0 \\
41 \cdot 0 \\
45 \cdot 0 \\
8 \cdot 0 \\
29 \cdot 0 \\
57 \cdot 0\end{array}$ & $\begin{array}{r}5 \cdot 2 \\
0 \\
8 \cdot 5 \\
4 \cdot 5 \\
25 \cdot 0 \\
24 \cdot 0 \\
18 \cdot 0 \\
24 \cdot 0 \\
7 \cdot 5 \\
14 \cdot 0 \\
9 \cdot 0 \\
10 \cdot 0 \\
2 \cdot 0 \\
7 \cdot 0 \\
8 \cdot 0 \\
4 \cdot 0 \\
1 \cdot 0\end{array}$ & $\begin{array}{r}3 \cdot 5 \\
0 \\
7 \cdot 0 \\
4 \cdot 0 \\
5 \cdot 5 \\
22 \cdot 0 \\
3 \cdot 0 \\
15 \cdot 0 \\
7 \cdot 0 \\
11 \cdot 0 \\
8 \cdot 0 \\
10 \cdot 0 \\
1 \cdot 0 \\
3 \cdot 0 \\
6 \cdot 0 \\
3 \cdot 0 \\
1 \cdot 0\end{array}$ & $\begin{array}{l}1 \cdot 7 \\
0 \\
1 \cdot 5 \\
0 \cdot 5 \\
19 \cdot 5 \\
2 \cdot 0 \\
15 \cdot 0 \\
9 \cdot 0 \\
0 \cdot 5 \\
3 \cdot 0 \\
1 \cdot 0 \\
0 \\
1 \cdot 0 \\
4 \cdot 0 \\
2 \cdot 0 \\
1 \cdot 0 \\
0\end{array}$ & $\begin{array}{c}12 \cdot 5 \\
0 \\
0 \\
0 \cdot 5 \\
2 \\
0 \\
13 \cdot 0 \\
0 \\
0 \cdot 5 \\
19 \cdot 0 \\
0 \\
7 \cdot 0 \\
39 \cdot 0 \\
37 \cdot 0 \\
0 \\
25 \cdot 0 \\
56 \cdot 0\end{array}$ & $\begin{array}{l}6 \cdot 2 \\
0 \\
0 \\
0 \cdot 5 \\
1 \cdot 0 \\
0 \\
0 \\
0 \\
0 \cdot 5 \\
4 \cdot 0 \\
0 \\
7 \cdot 0 \\
12 \cdot 0 \\
16 \cdot 0 \\
0 \\
5 \cdot 0 \\
55 \cdot 0\end{array}$ & $\begin{array}{c}6 \cdot 3 \\
0 \\
0 \\
0 \\
1 \cdot 0 \\
0 \\
13 \cdot 0 \\
0 \\
0 \\
15 \cdot 0 \\
0 \\
0 \\
21 \cdot 0 \\
21 \cdot 0 \\
0 \\
20 \cdot 0 \\
1 \cdot 0\end{array}$ & $\begin{array}{r}21 \\
0 \\
17 \\
8 \\
44 \\
30 \\
50 \\
37 \\
8 \\
65 \\
13 \\
34 \\
70 \\
47 \\
25 \\
75 \\
91\end{array}$ & $\begin{array}{r}20 \\
0 \\
5 \\
4 \\
28 \\
21 \\
38 \\
37 \\
11 \\
37 \\
13 \\
20 \\
47 \\
46 \\
3 \\
23 \\
66\end{array}$ & $\begin{array}{r}10 \\
0 \\
2 \\
3 \\
9 \\
21 \\
9 \\
4 \\
3 \\
7 \\
3 \\
3 \\
12 \\
41 \\
1 \\
3 \\
22\end{array}$ & $\begin{array}{l}14 \cdot 6 \\
19 \cdot 8 \\
22.6 \\
25 \cdot 0 \\
16 \cdot 0 \\
20 \cdot 5 \\
15 \cdot 6 \\
12 \cdot 0 \\
19 \cdot 5 \\
27 \cdot 0 \\
16 \cdot 3 \\
31 \cdot 6 \\
17 \cdot 5 \\
15 \cdot 4 \\
21 \cdot 0 \\
10 \cdot 9 \\
14 \cdot 4\end{array}$ & 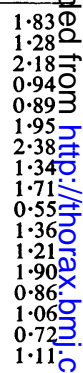 \\
\hline $\begin{array}{l}\text { Mean } \\
\text { SD }\end{array}$ & $\begin{array}{l}65 \cdot 3 \\
8 \cdot 63\end{array}$ & & $\begin{array}{r}46 \cdot 6 \\
9 \cdot 5\end{array}$ & $\begin{array}{l}3 \cdot 35 \\
0.6\end{array}$ & $\begin{array}{l}22 \cdot 5 \\
15 \cdot 7\end{array}$ & $\begin{array}{r}10 \cdot 1 \\
8 \cdot 1\end{array}$ & $\begin{array}{l}6 \cdot 5 \\
5 \cdot 6\end{array}$ & $\begin{array}{l}3 \cdot 6 \\
5 \cdot 6\end{array}$ & $\begin{array}{l}12 \cdot 4 \\
17 \cdot 2\end{array}$ & $\begin{array}{r}6 \cdot 3 \\
13 \cdot 4\end{array}$ & $\begin{array}{l}5 \cdot 7 \\
8 \cdot 4\end{array}$ & $\begin{array}{l}37 \cdot 3 \\
26 \cdot 2\end{array}$ & $\begin{array}{l}24 \cdot 6 \\
18 \cdot 4\end{array}$ & $\begin{array}{r}9 \cdot 0 \\
10 \cdot 4\end{array}$ & $\begin{array}{r}18 \cdot 8 \\
5 \cdot 4\end{array}$ & $\begin{array}{l}1.36 \frac{8}{3} \\
0.53\end{array}$ \\
\hline
\end{tabular}

For abbreviations see footnote to Table I.

three zones of the lung as compared to the nonfatal group. There was no significant difference between the bronchial mucous gland size in these two groups, but there was a significantly reduced proportion of small airways lumen in the lungs of the fatal group. A comparison of the mean values of the non-fatal group and the group without respiratory symptoms is also shown in Table VI. There was no significant difference between the right ventricular weights but the total amount of emphysema, the different types of emphysema, and the amount of emphysema in each zone were all significantly greater in the non-fatal group with respiratory symptoms. The bronchial mucous gland size was also significantly greater in this group than in the group without symptoms butit. there was no difference between the proportionfo of small airways lumen in these two groups.

The regional distribution of emphysema in the lungs was compared within each of the threes clinical groups. In the fatal group, zone 1 showe a mean of $71.1 \%$ of emphysema and zone $2 \%$ mean of $52.3 \%$. This was a significant difference of means $(P<0.05)$. A comparison of the mean amounts of emphysema in zone 2 and zone $\$$ $(32.8 \%)$ also showed a significant differences $(\mathrm{P}<0.05)$. In the non-fatal group with respiratori symptoms, zone 1 showed a mean of $39.4 \%$ em physema and zone 2 a mean of $24.7 \%$. This w not a significant difference $(\mathrm{P}>0.05)$. Zone 8 
T A B L E V I

COMPARISON OF MEAN VALUES FOR EACH OF THE THREE GROUPS ( $t$ test)

\begin{tabular}{|c|c|c|c|c|c|c|c|c|c|c|c|c|c|c|}
\hline \multirow{2}{*}{$\begin{array}{l}\text { Groups } \\
\text { Compared }\end{array}$} & \multirow{2}{*}{$\underset{\text { (g) }}{\text { RVW }}$} & \multirow{2}{*}{$\begin{array}{l}\text { LV/ } \\
\text { RV }\end{array}$} & \multirow{2}{*}{$\begin{array}{c}\text { Total } \\
\text { Emphy- } \\
\text { sema } \\
\%\end{array}$} & \multicolumn{3}{|c|}{$\begin{array}{l}\text { Centrilobular } \\
\text { Emphysema \% }\end{array}$} & \multicolumn{3}{|c|}{$\begin{array}{l}\text { Panlobular } \\
\text { Emphysema \% }\end{array}$} & \multicolumn{3}{|c|}{$\begin{array}{l}\text { Distribution of } \\
\text { Emphysema \% }\end{array}$} & \multirow{2}{*}{$\underset{\%}{\text { BMG }}$} & \multirow{2}{*}{$\mathbf{Q} \%$} \\
\hline & & & & Total & Dis & Des & Total & Dis & Des & Zone 1 & $\overline{\text { Zone } 2}$ & $\overline{\text { Zone } 3}$ & & \\
\hline $\begin{array}{l}\text { Fatal respiratory } \\
\text { disease } \\
\text { Non-fatal respiratory } \\
\text { disease } \\
t \\
P\end{array}$ & $\begin{array}{c}106 \cdot 2 \\
46 \cdot 6 \\
5 \cdot 64 \\
<0 \cdot 001\end{array}$ & $\begin{array}{r}1 \cdot 86 \\
3 \cdot 35 \\
6 \cdot 43 \\
<0 \cdot 001\end{array}$ & $\begin{array}{c}49 \cdot 3 \\
22 \cdot 5 \\
4 \cdot 19 \\
<0 \cdot 001\end{array}$ & $\begin{array}{c}11 \cdot 8 \\
10 \cdot 1 \\
0 \cdot 493 \\
>0 \cdot 60\end{array}$ & $\begin{array}{l}7 \cdot 1 \\
6 \cdot 5 \\
0 \cdot 260 \\
>0 \cdot 50\end{array}$ & $\begin{aligned} & 4 \cdot 7 \\
& 3 \cdot 6 \\
& 0 \cdot 541 \\
> & 0 \cdot 50\end{aligned}$ & $\begin{array}{c}38 \cdot 0 \\
12 \cdot 4 \\
3 \cdot 108 \\
<0 \cdot 01\end{array}$ & $\begin{array}{c}21.8 \\
6.3 \\
2.272 \\
<0.05\end{array}$ & $\begin{array}{c}16.1 \\
5.7 \\
1.891 \\
<0.10\end{array}$ & \begin{tabular}{|c|}
69.6 \\
$37 \cdot 3$ \\
$3 \cdot 783$ \\
$<0.001$
\end{tabular} & $\begin{array}{c}48 \cdot 7 \\
24 \cdot 6 \\
3.293 \\
<0.01\end{array}$ & $\begin{array}{c}32.0 \\
9.0 \\
3.508 \\
<0.01\end{array}$ & $\begin{array}{c}18 \cdot 8 \\
18 \cdot 8 \\
0 \cdot 007 \\
>0.90\end{array}$ & $\begin{array}{r}0.85 \\
1.36 \\
3.132 \\
<0.01\end{array}$ \\
\hline $\begin{array}{l}\text { Fatal respiratory } \\
\text { disease } \\
\text { No respiratory } \\
\text { symptoms } \\
t \\
\mathrm{P}\end{array}$ & $\begin{array}{c}106 \cdot 2 \\
42 \cdot 5 \\
5 \cdot 65 \\
<0 \cdot 001\end{array}$ & $\begin{array}{c}1 \cdot 86 \\
3 \cdot 75 \\
6 \cdot 32 \\
<0 \cdot 001\end{array}$ & \begin{tabular}{|c|}
$49 \cdot 3$ \\
$3 \cdot 1$ \\
$8 \cdot 30$ \\
$<0 \cdot 001$
\end{tabular} & $\begin{array}{c}11 \cdot 8 \\
2 \cdot 7 \\
2 \cdot 70 \\
<0.02\end{array}$ & $\begin{array}{c}7 \cdot 1 \\
2 \cdot 4 \\
2 \cdot 10 \\
<0 \cdot 05\end{array}$ & $\begin{array}{r}4 \cdot 7 \\
0 \cdot 3 \\
2 \cdot 46 \\
<0 \cdot 02\end{array}$ & $\begin{array}{c}38 \cdot 0 \\
0.3 \\
4 \cdot 96 \\
<0 \cdot 001\end{array}$ & $\begin{array}{r}21 \cdot 8 \\
0 \cdot 4 \\
3 \cdot 30 \\
<0 \cdot 01\end{array}$ & $\begin{array}{r}16 \cdot 1 \\
0.02 \\
2 \cdot 97 \\
<0.01\end{array}$ & $\begin{array}{r}69.6 \\
6.4 \\
9 \cdot 69 \\
<0.001\end{array}$ & $\begin{array}{c}48 \cdot 7 \\
1 \cdot 7 \\
7 \cdot 39 \\
<0 \cdot 001\end{array}$ & $\begin{aligned} & 32 \cdot 0 \\
& 0 \cdot 4 \\
& 4 \cdot 86 \\
&< 0 \cdot 001\end{aligned}$ & $\begin{array}{c}18 \cdot 8 \\
11 \cdot 9 \\
4 \cdot 65 \\
<0 \cdot 001\end{array}$ & $\begin{array}{r}0 \cdot 85 \\
1 \cdot 34 \\
3 \cdot 25 \\
<0 \cdot 01\end{array}$ \\
\hline $\begin{array}{l}\begin{array}{l}\text { Non-fatal respiratory } \\
\text { disease }\end{array} \\
\text { No respiratory } \\
\text { symptoms } \\
t \\
P\end{array}$ & $\begin{array}{c}46.6 \\
42.5 \\
1.82 \\
>0.05\end{array}$ & $\begin{array}{r}3 \cdot 35 \\
3 \cdot 75 \\
1 \cdot 39 \\
>0 \cdot 10\end{array}$ & $\begin{array}{c}22 \cdot 5 \\
3 \cdot 1 \\
4 \cdot 65 \\
<0 \cdot 001\end{array}$ & $\begin{array}{c}10.1 \\
2.7 \\
3.20 \\
<0.01\end{array}$ & $\begin{array}{c}6.5 \\
2.4 \\
2.44 \\
<0.05\end{array}$ & $\begin{array}{r}3 \cdot 6 \\
0 \cdot 3 \\
2 \cdot 13 \\
<0.05\end{array}$ & $\begin{array}{c}12.4 \\
0.3 \\
2.71 \\
<0.02\end{array}$ & $\begin{array}{r}6.3 \\
0.4 \\
1.69 \\
>0.05\end{array}$ & $\begin{array}{r}5.7 \\
0.02 \\
2.61 \\
<0.02\end{array}$ & $\begin{array}{c}37 \cdot 3 \\
6.4 \\
4 \cdot 39 \\
<0.001\end{array}$ & $\begin{array}{c}24 \cdot 6 \\
1.7 \\
4 \cdot 72 \\
<0.001\end{array}$ & $\begin{array}{c}9.0 \\
0.4 \\
3.18 \\
<0.01\end{array}$ & \begin{tabular}{|c|}
18.8 \\
11.9 \\
4.19 \\
$<0.001$
\end{tabular} & $\begin{array}{l}1.35 \\
1.34 \\
0.116 \\
>0.90\end{array}$ \\
\hline
\end{tabular}

For abbreviations see footnote to Table I.

showed $10.8 \%$ of emphysema and this was significantly different from the mean of zone 2 $(P=0.01)$. The amounts of emphysema in the group without respiratory symptoms were too small for these comparisons to be significant.

Each case was classified according to the predominant type of emphysema present, and amounts of less than $5 \%$ of a different type of emphysema were included with the major type (Hicken et al., 1966). Cases with more than $5 \%$ of both types of emphysema were regarded as 'mixed' emphysema. In the fatal group there were four patients with centrilobular emphysema, eight with panlobular emphysema, and six with mixed emphysema. The non-fatal group showed eight patients with centrilobular emphysema, three with panlobular emphysema, and five with mixed emphysema. In the group without respiratory symptoms there were eight patients with slight centrilobular emphysema and one with mild panlobular emphysema. The patients in the fatal and non-fatal groups together represented 12 cases of centrilobular emphysema, 13 of panlobular emphysema, and nine of mixed emphysema. The mean right ventricular weight for each of these groups was calculated and the centrilobular emphysema group had a mean of $58.2 \mathrm{~g}$, the panlobular emphysema group a mean of $93.3 \mathrm{~g}$, and the mixed emphysema group a mean of $84.0 \mathrm{~g}$. There was no significant difference between any of these means.

\section{DISCUSSION}

This study has shown that there is a relationship between the amount of the lung replaced by em- physema and the clinical severity of respiratory disease. The patients dying from chronic airways obstruction had an average of $49.3 \%$ emphysema, the group with non-fatal respiratory disease had $22.5 \%$ emphysema, and the group without respiratory symptoms had $3.1 \%$. These findings are similar to those of Sweet et al. (1961), who found that the average amount of lung involved by emphysema, in those dying from it, was $54 \%$, and in a group where symptoms were present but emphysema did not contribute to death, the average amount was $20 \%$. Their conclusions that emphysema causes symptoms when $20 \%$ of the lung is involved and causes death when approximately $50 \%$ is involved by it are supported by the present series. Mitchell et al. (1966) state that, as a general rule, the more severe the emphysema, the more severe the clinical state of the patient and the more likely is death from complications of the disease. They did note that there were exceptions to this rule and these have also been found in this series. Four patients in the fatal group had relatively small amounts of emphysema (cases 15,21 , 34 , and 49) but three of these patients $(15,34$, and 49) had very small proportions of small airways lumen in the lung $(0.57 \%, 0.61 \%$, and $0.49 \%$ respectively). The combined effect of loss of small airways and reduction in alveolar surface area may be important in these cases. One patient in the non-fatal group with respiratory symptoms (69) had emphysema involving more than 50\% of the lung and did not show a very marked reduction in the proportion of small airways lumen $(1 \cdot 11 \%)$. Pratt and Kilburn (1970), using postmortem ventilatory function tests, found a reduc- 
tion in expiratory volume only when $30 \%$ of the lung was involved by emphysema, and this correlates well with the average of $22.5 \%$ of lung involved in the group with symptoms of chest disease who had not developed complications of emphysema in the present study. Not all series have shown this relationship between the amount of emphysema and the clinical state of the patient: Cullen et al. (1970) found the incidence of right heart failure to be unrelated to total emphysema score.

This study has also shown a significant correlation between the amount of emphysema in the lung and the right ventricular weight. Previous studies have produced conflicting evidence on this relationship. Burrows et al. (1966) found no relationship between these two parameters, and Cromie (1961) found an inverse correlation between them. Both these studies, however, were based on measurements of the thickness of the right ventricle and not on the weight. Cullen et al. (1970) showed that there was significant thinning of the right ventricle, in obstructive lung disease, but the endocardial surface area was increased. Measurements of myocardial thickness cannot therefore be accepted as evidence of right ventricular hypertrophy. Wyatt, Fischer, and Sweet (1964) and Foraker, Bedrossian, and Anderson (1970) found a relationship between the incidence of severe emphysema and right ventricular hypertrophy, and this is confirmed in the present study. However, Hicken et al. (1966), Bignon et al. (1969), and Hasleton (1973) found no simple relationship between right ventricular weight and the total percentage of abnormal air space in the lung. It may be that the inclusion in the present series of a whole spectrum of disease states from the normal to end stage disease may have contributed to the finding of a positive relationship between right ventricular weight and the amount of emphysema in the lung. This study has shown no significant relationship between the amount of centrilobular emphysema in the lung and either the clinical state of the patient or the right ventricular weight. However, there was a relationship between the amount of panlobular emphysema and right ventricular weight. Patients dying from chronic airways obstruction may have either centrilobular emphysema, panlobular emphysema, or mixed emphysema, but only panlobular emphysema gave a significant quantitative correlation with right ventricular weight. Leopold and Gough (1957), Dunnill (1961), Hicken et al. (1966), and Bignon et al. (1970) found centrilobular emphysema more important than panlobular emphysema in causing right ventricular hypertrophy. Hicken et al. (1966) and Hasleton (1973), who found nof relationship between any type of emphysema ande. right ventricular weight, grouped their cases of emphysema according to the predominant type present and then compared the ventricular weights ${ }^{\circ}$ in the three groups - centrilobular emphysema, $\vec{O}$ panlobular emphysema, and mixed emphysema. $A-$ similar grouping performed in the present study. also failed to show any relationship between the type of emphysema and right ventricular weight $x$ This method would appear to have several dis $-\omega$ advantages as it provides no comparison between the type of emphysema and the clinical state of the patient nor does it relate the amount of each type of emphysema to the right ventricular weight. Many cases are put into a 'mixed' group which' thus provides little useful information about the varying proportions of each type of emphysemav present in the lung. Several studies have shown panlobular emphysema to be more important thane centrilobular emphysema in causing right ventriō cular hypertrophy. Both Sweet et al. (1961) and Wyatt et al. (1964) found this, and Wyatt, Fischer and Sweet (1962), whose cases were all of pan lobular emphysema, found a positive correlation between increasing severity of emphysema and right ventricular hypertrophy. The findings in the present study are in agreement with these and also that of James (1966), who found no relation between right ventricular weight and the amoun of emphysema, but 30 of his 31 cases were of centrilobular emphysema. Using point-counting techniques, panlobular emphysema gives largen numbers than centrilobular emphysema (Thurl:beck, 1968); this may be an important factor i the establishment of a quantitative correlation between panlobular emphysema and right ventri cular weight but not between centrilobular emo physema and right ventricular weight. Few studies have been carried out on the regional distributio of emphysema throughout the lung, and none has been done on the relationship between distribution of emphysema and right ventricular weight. The blood flow through the lung is not uniform in all areas but decreases steadily from the base to the apex (West et al., 1968). It may be assumed from this that emphysema involving the lower region of the lung would affect the ventilation/perfusions ratio more than if it involved the upper regions.o West et al. (1968) using radioactive carbon dioxide and xenon were able to divide the lung into three zones by the relative magnitude of the pulmone ary arterial, venous, and alveolar pressures. Three similar zones were used in the present study to 
determine the effect of different distributions of emphysema on the right ventricle, and it has been shown that emphysema increased significantly in each of the three zones as disability increased. Also there was a significant correlation between increasing amounts of emphysema in each zone and right ventricular weight. Within the fatal group there was a significant decrease in the amount of emphysema from apex to the base but this was not marked in the other two groups. Different distributions of emphysema, depending on whether it was centrilobular emphysema or panlobular emphysema, have been found by Bignon et al. (1969, 1970) but Thurlbeck (1963) found panlobular emphysema to be evenly distributed in the lung. Greenberg, Boushy, and Jenkins (1966), measuring lobar distribution, found that in mild or moderate emphysema there was more disease in the upper as compared to the middle or lower lobes, but when involvement of the lung exceeded $50 \%$ interlobar differences tended to disappear. In this study, where a zonal distribution was measured, emphysema was always more severe at the apex than in the middle and lower zones, no matter the degree of clinical disability. Emphysema would also appear to increase in each area of the lung, as the total amount increases, rather than in any one area.

The size of the bronchial mucous glands has been shown to correlate well with clinical symptoms of chronic bronchitis (Scott, 1973). In this study the mean bronchial mucous gland size was significantly greater in those with symptoms than in those without them, but there was no difference between the mean sizes of the fatal and non-fatal respiratory disease groups. Also there was no correlation between the size of the bronchial mucous glands and right ventricular weight. Hentel et al. (1963) stated that chronic bronchitis without emphysema could cause right heart failure but no such cases were found in the present study. Millard (1967) suggested that a relationship might exist between bronchial gland size and right ventricular hypertrophy but the present study would confirm the findings of Dunnill (1968b), who found no simple relationship between the right ventricular weight and bronchial gland size. No correlation was found between the amount of emphysema and the size of the bronchial mucous glands. This confirms the results of Dunnill (1968b), Cullen et al. (1970), and Ryder, Dunnill, and Anderson (1971). In the present study the proportion of the lung occupied by the lumen of small airways (less than $2 \mathrm{~mm}$ diameter) was significantly reduced in patients who died from chronic airways obstruction as compared to the non-fatal and symptom-free groups. Also there was an inverse correlation between the proportion of small airways lumen in the lung and right ventricular weight. Bignon et al. (1968) found that right ventricular hypertrophy was related to disseminated bronchiolostenosis, and also Bignon et al. (1970) found a slight correlation between right ventricular weight and the percentage of bronchioles of less than $350 \mu$ diameter. In the present study the reduction in the proportion of small airways lumen in the lung was also found to be related to the amount of emphysema in the lung. Leopold and Gough (1957) found a reduction in supplying bronchioles in centrilobular emphysema, and Matsuba and Thurlbeck (1972) also found a reduction in airways of less than $2 \mathrm{~mm}$ diameter in emphysematous lungs. The values they obtained are very similar to those in the present study-0.0142 $(1.42 \%)$ for the non-emphysematous lungs and $0.0087(0.87 \%)$ for emphysematous lungs. In this study the non-fatal and the symptom-free patients had mean values of $1.36 \%$ and $1.34 \%$ respectively whereas the fatal group had a mean value of $0.85 \%$. Matsuba and Thurlbeck (1972) do not make clear the extent of the emphysema or clinical disability in their series. The relationship between loss of small airways and death from airways obstruction has also been shown by post-mortem tantalum bronchography by Scott and Steiner (1975). The relationship between the reduction in small airways lumen and the different types of emphysema in this study is of interest. There was a significant inverse correlation between panlobular emphysema and the proportion of small airways lumen in the lung but no such relationship with centrilobular emphysema. As panlobular emphysema affects a much larger proportion of the lung lobule than centrilobular emphysema this may explain why small airways occupy less of the lung parenchyma since it has already been shown that the more emphysema present in the lung, of both types, the smaller the proportion of small airways lumen becomes.

My thanks are due to Dr. P. Howard and Dr. G. H. Roberts for helpful advice and criticism.

\section{REFERENCES}

Bignon, J., Andre-Bougaran, J., and Brouet, G. (1970). Parenchymal, bronchiolar, and bronchial measurements in centrilobular emphysema. Thorax, 25, 556.

Khoury, F., Even, P., André, J. and Brouet, G. (1968). Etude anatomique quantitative des bron- 
chopneumopathies chroniques obstructives. Résultats préliminaires. Individualisation de formes avec bronchiolostenoses disséminées. Revue de Tuberculose et de Pneumologie, 32, 207.

- - , - - - and - (1969). Morphometric study in chronic obstructive bronchopulmonary disease. American Review of Respiratory Disease, 99, 669.

Burrows, B., Fletcher, C. M., Heard, B. E., Jones, N. L., and Wootliff, J. S. (1966). The emphysematous and bronchial types of chronic airways obstruction. Lancet, 1, 830.

Cromie, J. B. (1961). Correlation of anatomic pulmonary emphysema and right ventricular hypertrophy. American Review of Respiratory Disease, 84, 657.

Cullen, J. H., Kaemmerlen, J. T., Daoud, A., and Katz, H. L. (1970). A prospective clinical-pathologic study of the lungs and heart in chronic obstructive lung disease. American Review of Respiratory Disease, 102, 190.

Dunnill, M. S. (1961). An assessment of the anatomical factor in cor pulmonale in emphysema. Jour nal of Clinical Pathology, 14, 246.

(1962). Quantitative methods in the study of pulmonary pathology. Thorax, 17, 320.

(1968a). Quantitative methods in histology. In Recent Advances in Clinical Pathology, p. 405. Series V, edited by S. C. Dyke. J. \& A. Churchill, London.

(1968b). Morphometry of the human lung in health and disease. In Form and Function in the Human Lung, p. 24. Edited by G. Cumming and L. B. Hunt. E. \& S. Livingstone, Edinburgh and London.

-, Massarella, G. R., and Anderson, J. A. (1969). A comparison of the quantitative anatomy of the bronchi in normal subjects, in status asthmaticus, in chronic bronchitis, and emphysema. Thorax, 24, 176.

Foraker, A. G., Bedrossian, C. W. M., and Anderson, A. E. Jr. (1970). Myocardial dimensions and proportions in pulmonary emphysema. Archives of Pathology, 90, 344.

Fulton, R. M., Hutchinson, E. C., and Jones, A. M. (1952). Ventricular weight in cardiac hypertrophy. British Heart Journal, 14, 413.

Greenberg, S. D., Boushy, S. F., and Jenkins, D. E. (1966). Differences in the lobar distribution of emphysema and chronic bronchitis in the lung. In Current Research in Chronic Airways Obstruction, p. 101. US Department of Health, Education and Welfare, Virginia.

Hasleton, P. S. (1973). Right ventricular hypertrophy in emphysema. Journal of Pathology, 110, 27.

Heard, B. E. (1958). A pathological study of emphysema of the lungs with chronic bronchitis. Thorax, 13, 136.

(1969). Pathology of Chronic Bronchitis and Emphysema, p. 8. Churchill, London.

Hentel, W., Longfield, A. N., Vincent, T. N., Filley, G. F., and Mitchell, R. S. (1963). Fatal chronic bronchitis. American Review of Respiratory Disease, 87, 216.
Hicken, P., Heath, D., and Brewer, D. (1966). The relation between the weight of the right ventricle and the percentage of abnormal air space in the lung in emphysema. Journal of Pathology and $\frac{\omega}{\partial}$ Bacteriology, 92, 519.

James, W. R. L. (1966). The right ventricle and pulmonary vascular obstruction in bronchitis with emphysema. British Heart Journal, 28, 512.

Leopold, J. G. and Gough, J. (1957). The centri-ت lobular form of hypertrophic emphysema and its relation to chronic bronchitis. Thorax, 12, 219.

Matsuba, K. and Thurlbeck, W. M. (1971). The number and dimensions of small airways in non- $\underline{\omega}$ emphysematous lungs. American Review of Respiratory Disease, 104, 516.

_- and - (1972). The number and dimensions of small airways in emphysematous lungs. American Journal of Pathology, 67, 265.

Millard, J. (1967). Pulmonary hypertension in chronico bronchitis. (Abstr.) Thorax, 22, 286.

Mitchell, R. S., Silvers, G. W., Dart, G. A., Petty, صِ T. L., Vincent, T. N., Ryan, S. F., and Filley, G. F. (1966). Clinical and morphologic correla- $\overrightarrow{0}$ tions in chronic airway obstruction. In Current Research in Chronic Airways Obstruction, p. 109. US Department of Health, Education and Welfare, Virginia.

Pratt, P. C. and Kilburn, K. H. (1970). A modern concept of the emphysemas based on correlationson of structure and function. Human Pathology, 120 443.

Restrepo, G. and Heard, B. E. (1963). The size of the $\overline{\overline{0}}$ bronchial glands in chronic bronchitis. Journal of 3 Pathology and Bacteriology, 85, 305.

Ryder, R. C., Dunnill, M. S., and Anderson, J. A. (1971). A quantitative study of bronchial mucous gland volume, emphysema and smoking in at necropsy population. Journal of Pathology, 104, 59.

Scott, K. W. M. (1973). An autopsy study of bronchial mucous gland hypertrophy in Glasgow. American: Review of Respiratory Disease, 107, 239.

and Steiner, G. M. (1975). Post mortem assessment of chronic airways obstruction by tantalum? bronchography. Thorax, 30, 405.

Sweet, H. C., Wyatt, J. P., Fritsch, A. J., and Kinsella, P. W. (1961). Panlobular and centrilobular emphysema. Correlation of clinical findings with pathologic patterns. Annals of Internal Medicine, $55,565$.

Thurlbeck, W. M. (1963). The incidence of pulmon- $-\omega$ ary emphysema. American Review of Respiratory Disease, 87, 206.

(1968). Discussion on morphometry of the human lung. In Form and Function in the Humans Lung, edited by G. Cumming and L. B. Hunt, pp. 37 and 38. Livingstone, Edinburgh and London.

West, J. B., Glazier, J. B., Hughes, J. M. B., and Maloney, J. E. (1968). Recent work on the distribution of pulmonary blood flow and topographical differences in alveolar size. In Form and Function in the Human Lung, edited by G. 
Cumming and L. B. Hunt, p. 111. Livingstone, Edinburgh and London.

Wyatt, J. P., Fischer, V. W., and Sweet, H. C. (1962). Panlobular emphysema: anatomy and pathodynamics. Diseases of the Chest, 41, 239. (1964). The pathomorphology of the emphysema complex. Part 1. American Review of Respiratory Disease, 89, 533.

Requests for reprints to: Dr. K. W. M. Scott, Department of "Pathology, The Royal Hospital, Wolverhampton. 